\title{
Does stage of illness influence recovery- focused outcomes after psychological treatment in bipolar disorder? A systematic review protocol
}

\author{
Hailey Tremain $^{1^{*}}$ (D), Kathryn Fletcher ${ }^{1}$, Jan Scott ${ }^{1,2}$, Carla McEnery ${ }^{1,4,5}$, Michael Berk ${ }^{3,4,5,6}$ and Greg Murray ${ }^{1}$
}

\begin{abstract}
Background: There is great interest in the possibility that 'stage of illness' moderates treatment outcomes in bipolar disorder (BD). Much remains unknown about the construct of stage of illness, but there is evidence that effectiveness of psychosocial interventions may depend on factors that are plausible proxy measures of stage of illness (e.g., number of episodes). To date, reviews of this data have focused solely on clinical outcomes (particularly symptoms and relapse rates), but a range of recovery-focused outcomes (including functioning, cognitive functioning, and quality of life) have been measured in individuals with established BD. The aim of the proposed systematic review is to synthesise existing evidence for plausible proxy measures of stage of illness as moderators of recovery-focused and functional outcomes in psychosocial treatment studies of BD.
\end{abstract}

Methods: The proposed review will follow PRISMA guidelines; Scopus, PsychINFO, PubMed and Web of Science will be searched for empirical studies of psychosocial interventions used for established (clinical stages 2-4) BD; and findings will be summarised in a narrative synthesis of clinical stage of illness (operationalised in proxy measures identified in existing staging models) as a moderator of recovery-focused and functional outcomes of psychosocial interventions for established bipolar disorder.

Discussion: This review will contribute to the literature by expanding upon previous reviews and potentially inform the psychosocial treatment of established BD. Implications include assisting clinicians, consumers and researchers to identify and select interventions most appropriate to recovery-focused goals based on individuals' clinical status.

Systematic review registration: PROSPERO CRD42016037868

Keywords: Bipolar disorder, Psychosocial interventions, Psychotherapy, Treatment, Moderator, Clinical staging, Stage of illness, Recovery, Functioning, Quality of life

\section{Background}

A developing body of research suggests that bipolar disorder (BD) may be characterised by progressive changes over time, such that the clinical and functional features experienced differ in those earlier versus later in the course of illness [1-5]. Most importantly, it has been argued that the notion of 'stage of illness' has potential to generate long-awaited improvements in outcomes for

\footnotetext{
* Correspondence: htremain@swin.edu.au

${ }^{1}$ Centre for Mental Health, Faculty of Health Arts and Design, Swinburne University, PO Box 218, John St Hawthorn VIC, Melbourne 3122, Australia Full list of author information is available at the end of the article
}

those with $\mathrm{BD}$, via tailoring of treatments to particular stages of illness $[4,6,7]$. Empirical research into staging of illness in BD is in its infancy, with existing studies limited in important ways. Below, we briefly review what is known about staging of illness in the psychosocial treatment of BD and identify key gaps to be addressed by the proposed systematic review.

\section{Staging models}

Several (largely compatible) clinical staging models have been described to capture the key features of BD within putative stages of illness. These broadly refer to an initial 
asymptomatic at-risk stage, followed by a stage characterised by non-specific symptoms and then a stage with more specific mood disorder-related, but subsyndromal symptoms. A syndromal stage, usually referred to as clinical stage 2 then follows, wherein mood episodes meet recognised diagnostic criteria and functional impacts begin to emerge, followed by stage 3 , where a repeated pattern of recurrences and relapses is common. The final or end stage (stage 4) is characterised by chronicity manifested by persistent or highly recurrent symptoms and progressively severe functional impacts $[8,9]$. Berk and colleagues [8] highlight the role of accumulating mood episodes and associated functional impairments. A convergent (yet distinct) model proposed by Kapczinski and colleagues [9] primarily focuses on inter-episode functional and cognitive decrements as BD progresses. This model only addresses post-onset threshold disorder and does not detail the subsyndromal phases of BD.

Models of clinical staging in BD remain largely theoretical: no model has been subjected to systematic empirical testing. Stage of illness is conceptualised as multidimensional and multisystemic, encapsulating more than severity. Severity, characterised by linear and unidimensional changes (increasingly more severe symptoms or more frequent episodes) may represent one factor which changes between stages of illness [10]. However, additional related features are hypothesised to distinguish stages of illness, reflecting progression and extension of disease: medical and psychiatric comorbidities, cognitive decrements, reduced response to treatment and psychosocial sequelae (such as loss of autonomy and social functioning), although the boundaries of stages remain undefined [11]. Such stages could, in future, be distinguished by biomarkers. However, key questions remain around biological markers of this proposed multisystemic, pathophysiological progression and, critically, the proportion of individuals who experience progression through each stage of illness [10, 12]. Nonetheless, the International Society for Bipolar Disorders Taskforce for Staging has concluded that sufficient evidence has accumulated to support the use of staging of illness within established BD (i.e., post-onset or full-threshold disorder), which we will refer to as clinical stages 2-4. Also, the task force notes that such a distinction is likely to be of heuristic value in the treatment of BD [13]. Likewise, data-driven approaches have begun to distinguish broad post-onset groups based on features such as functioning, number of episodes, and illness duration, and these groups show different responses to treatment $[5,14,15]$. There is also some evidence that neuroimaging and cognition vary by stage of illness and emerging evidence of biomarkers that may track illness trajectory $[15,16]$.

\section{Benefits of illness staging for bipolar disorder}

A validated staging model for bipolar disorder therefore holds significant, but as yet unrealised, promise. A validated model for illness stages 0 and 1 would provide the basis for developing preventive strategies and interventions which may delay the onset of $\mathrm{BD}$ and circumvent associated morbidity [10, 17], an approach which has shown promise in other serious mental disorders [18]. Identification of factors that arrest progression to the later, more debilitating stages of illness has potential to reduce loss of functioning, morbidity, mortality and treatment resistance $[4,8]$. Developing validly stage-tailored interventions could ensure optimally effective psychological and pharmacological interventions are implicated for individuals at their specific illness stage $[2,7,19]$. Of particular relevance here, the stage of illness heuristic may also help clinicians and consumers come to a shared understanding of the most meaningful targets for interventions. For example, a focus on managing risk factors may benefit the asymptomatic at-risk group (in psychiatry, this group is only recognisable as those with a family history of a severe mental disorder), but low risk, non-pharmacological interventions would be most realistic for this group. Relief of symptoms and management of triggers may have greater impact for those at stages associated with more recent evolution of established illness (primarily stage 2), whereas the quality of life or functioning may be more meaningful intervention targets in stage 4 and for some in stage $3[19$, 20]. In later stages of illness, it is increasingly likely that combined psychopharmacological approaches will be employed, with increasing acknowledgement that psychological interventions are important in end-stage BD [21].

\section{Proxy measures of stage in established BD}

In the absence of robust evidence for biomarkers and biobehavioural boundaries of the putative stages in $\mathrm{BD}$, empirical research has relied on proxies to operationalise stage of illness. These have not been widely employed in subthreshold or asymptomatic stages of illness (i.e., stages 0 or 1 ), but have been increasingly considered in established BD. Most commonly, lifetime number of mood episodes has been used [22-27]. While a potentially useful and plausible proxy measure, ${ }^{1}$ number of episodes alone cannot fully capture the complexity of the proposed staging phenomenon $[4,9]$, nor the notion of 'disease extension, which is a fundamental component of medical staging models (e.g., a tumour spreads to lymph nodes, then to other bodily systems) [28]. It has been proposed that analogues of physical disease extension in the case of mental disorders may be comorbidities, functioning or disability, and cognitive functioning [2, 5, 9, 29]. For example, measures such as the Functional Assessment Short Test (FAST) or Social and Occupational Functioning Assessment Scale (SOFAS) differentiate between putative 
stages of established BD [5, 9, 29, 30]. Each of these plausible proxy measures has limitations, for example the circularity inherent in considering functioning at baseline as a predictor of functioning post-intervention and issues with equating number or type of comorbidities to severity or progression. Based on an extensive review of published models and theories of staging, the proxy measures for stage of illness recognised in this review, are outlined in Table 1.

Most research to date has relied upon the number of episodes as the proxy measure of stage of illness, while recognising that other clinical factors are relevant to stage of illness, and as such, these additional variables will be included as exploratory additional plausible proxy measures. Given the identified limitations of such proxies, all conclusions will consider these limitations and where available, change from premorbid functioning or cognition will be utilised. Other clinical course factors certainly have relevance to staging models, including age at onset, duration of illness, bipolar type and predominant polarity $[16,31]$. However, due to lack of consistency in how these feature across staging proposals, this review will examine those potential proxy measures with most support to date.

\section{Psychosocial interventions and staging of illness}

Research into staging of illness is in its infancy, with many questions remaining. Research to date suggests that both pharmacotherapy and adjunctive psychotherapy are more effective for some individuals with $\mathrm{BD}$ than for others, providing some support for the hypothesis that stage of illness may influence response to therapy or outcomes [19, 25, 32, 33]. However, evidence forwarded to support the assertion that stage of illness may moderate outcomes in BD rests almost entirely on post hoc statistical analyses of outcomes in clinical trials not designed to address the staging hypothesis.

An influential finding in this literature arose in post hoc analyses of a pragmatic effectiveness trial of cognitive-behaviour therapy (CBT) for BD [34] which demonstrated that CBT was superior to treatment as usual (TAU) alone in improving outcomes for individuals with 11 or fewer lifetime mood episodes, with no benefits of adding CBT to

Table 1 Plausible proxy measures for stage of illness relevant to this review

\begin{tabular}{l}
\hline Variables \\
\hline 1. Number of mood episodes [22-26], with consideration by episode \\
type and of frequency where available \\
2. Functioning/disability $[5,9,29,30]$ \\
3. Cognitive functioning/cognitive impairment/neurocognitive \\
deficit $[2,5,9,29]$ \\
4. Comorbidities [9]
\end{tabular}

TAU in those with more than 11 episodes. Similar findings have been reported in several psychoeducation trials, wherein those retrospectively classified within earlier stages of established illness (using number of episodes or functioning as proxy measures) were more likely to report benefits than those retrospectively classified into later stages of illness [22, 30, 35, 36]. Findings from the psychotherapy arm of the large-scale Systematic Treatment Enhancement Program for Bipolar Disorder (STEP-BD) trial align with this; participants with fewer lifetime manic and depressive episodes were faster and more likely to respond to psychotherapy overall, especially lower intensity interventions $[14,37]$. Extrapolating from studies to date is consistent with the staging hypothesis of $\mathrm{BD}$, insomuch as psychological interventions may be generally less effective as BD advances.

There is growing recognition that non-clinical, recoveryfocused outcomes are valued by consumers and that these are distinct from yet complementary to symptom-based intervention targets [20,38]. These may include functional outcomes, such as social functioning including autonomy, occupational functioning, and interpersonal functioning and cognitive functioning including memory, learning and attention, and quality of life outcomes. For the present purposes, it is noteworthy that these outcomes have been argued to be particularly relevant to individuals with later stage established BD (stages 3-4): Berk and colleagues propose that as $\mathrm{BD}$ progresses, symptom remission may be a less important target for intervention, and recovery-focused outcomes such as improved quality of life and daily functioning may warrant higher priority [19].

A novel and complex hypothesis therefore emerges from this literature. As argued by Murray et al. [20], different outcomes may become more relevant as $\mathrm{BD}$ progresses, and different interventions may be best positioned to target these outcomes. For instance, Torrent and colleagues compared TAU pharmacotherapy with intensive psychoeducation and functional remediation in a sample of individuals with significant functional impairment and a large number of previous mood episodes, consistent with the later putative stages of $\mathrm{BD}[39,40]$. Participants in the functional remediation group reported greater improvements in functioning (particularly autonomy) than those receiving psychoeducation or TAU, and these differences reached significance at 12-month follow-up. Conversely, no significant differences in relapses or residual depressive symptoms were reported between groups at follow-up. These findings may suggest differential effectiveness of two interventions for those considered at the later stages of BD for improving functioning, but not symptoms. A recent pilot study of a mindfulness intervention targeted individuals with late-stage $\mathrm{BD}$, based on number of episodes [32]. Moderate to large improvements were reported in participants' quality of life, while symptom change was 
non-significant. While limited, this study encourages further research into the hypothesis that different outcomes may become relevant for individuals at later illness stages. Conversely, several psychoeducation trials with samples considered in the later stages of illness (based on functioning or number of episodes) have failed to find significant improvements in symptoms or recoveryfocused outcomes, including quality of life and functioning. Taken together these findings may suggest that different interventions and different targets may be required as BD progresses [39-42].

Pharmacotherapy is the first-line treatment for bipolar disorder, with high-quality evidence supporting the effectiveness of medications for mania and depression in both the acute and maintenance phases (e.g., [43-45]). However, areas of uncertainty remain, such as effective options for addressing treatment resistance and refractory depression [45]. A number of systematic reviews and meta-analyses conducted in recent years support the effectiveness of psychosocial interventions as adjuncts to medication in the treatment of bipolar disorder [33, 46-48]. Overall, medium effect-size benefits have been found for outcomes including manic and depressive relapse, residual symptoms and medication adherence. All such reviews note significant limitations within the evidence base, including the small number of studies, heterogeneous assessment of outcomes, and limited study quality [49]. Several reviews have noted that factors relating to stage of illness (including the proxy measures examined in the proposed review) may partially moderate the effectiveness of these interventions [33, 47, 48], especially CBT and psychoeducation.

To the best of our knowledge, only two studies have directly reviewed plausible proxy measures of stage of illness as moderators of psychosocial interventions for BD. A meta-analysis of psychotherapy studies for BD aimed to assess whether number of lifetime mood episodes predicts response to psychotherapy [50]. This study did not find evidence for an impact of number of episodes in the effectiveness of psychological therapies for preventing relapse in BD. However, due to the methodological homogeneity required for meta-regression, only six studies were included, with the single outcome variable of relapse rates. This narrow scope excluded important findings in the literature, in particular those from the largest randomised controlled trial (RCT) of CBT for BD to date (above; excluded due to categorical recording of number of episodes) [34]. More recently, a narrative synthesis of the role of accumulating episodes was undertaken [25], concluding that response to psychotherapy declines with accumulating mood episodes. However, this review was confined to studies reporting on a direct comparison between those classified as being within early versus late stages of established BD; thus, only one RCT of group psychoeducation and one CBT trial were included. Furthermore, only symptom-focused outcomes (relapse rates and time to recurrence) were examined.

The limited number of outcomes examined in prior reviews has confined the exploration of the complexity and applications of staging of illness, disallowing conclusions to be drawn as to whether different outcomes may be more effective treatment foci at different stages of illness. Another key limitation of existing reviews is the use of a single plausible proxy measure, lifetime number of mood episodes [12, 51]. Further, both relied on post hoc analyses to draw conclusions, due to the dearth of studies explicitly seeking to explore stage of illness or plausible proxy measures thereof as a moderator of outcomes.

\section{The proposed systematic review}

A comprehensive systematic review is now required which attends to (i) recovery-focused and functional outcome measures and (ii) an expanded range of plausible proxy measures of stage of illness in post-onset $\mathrm{BD}$, derived from a combination of empirical data and prominent theoretical models. The proposed review will follow PRISMA guidelines and provide a synthesis of the evidence for stage-based moderation (as operationalised in plausible proxy-based moderation) of psychosocial interventions for recovery-focused outcomes in established $\mathrm{BD}$ and identify key priorities for further research.

The overarching aim of the planned review is to advance understanding of the notion of stage of illness in BD, by retrospectively interrogating findings related to psychosocial interventions' impact on recovery-focused and functional outcomes in stages 2-4 of BD. Specifically, the objectives are to explore whether plausible proxy measures of stage of illness may moderate recovery-focused intervention outcomes in $\mathrm{BD}$ and whether there is any evidence that this is associated with specific types of intervention. It is expected that this review will answer two key questions regarding the status of the staging construct in $\mathrm{BD}$ : (i) is there any evidence that plausible proxy measures of stage moderate recovery-focused outcomes in adjunctive psychosocial treatment of established BD? (ii) If so, is the effect more strongly associated with particular interventions?

\section{Methods}

The systematic review has been registered with the International Prospective Register of Systematic Reviews (PROSPERO, http://www.crd.york.ac.uk/PROSPERO, registration number: CRD42016037868). It will be conducted in accordance with Preferred Reporting Items for Systematic Reviews and Meta-Analyses Protocol (PRISMA-P) recommendations for systematic review protocols [52] (see Additional file 1), and the findings will be reported using the PRISMA guidelines [53]. 


\section{Criteria for study inclusion Population}

Included studies will be empirical and comprise participants aged 18 and over with a BD I, BD II, BD-NOS, not elsewhere classifiable, or other specified BD diagnosis (meeting internationally recognised diagnostic criteria as in the DSM-IV or 5, or ICD-10), without restrictions based on characteristics such as psychosis, comorbidity or mood status at study entry. Studies with participants under 18 years old have been excluded due to the additional complexity in diagnosis and treatment in this population, as well as potential divergent trajectory and phenomenology of early-onset or paediatric BD [54]. Studies with participants with other diagnoses (specifically schizophrenia and schizoaffective disorder) will be excluded unless these reports provide separate analyses relating to those with diagnosed $\mathrm{BD}$.

\section{Interventions}

Studies will detail the outcomes of any psychological or psychosocial intervention or combination of psychosocial interventions (any non-medical intervention intended to modify symptoms, behaviour, emotional state, or feelings) for $\mathrm{BD}$, including (but not restricted to) cognitivebehavioural therapy, mindfulness-based cognitive therapy, interpersonal and social rhythm therapy, psychoeducation, family-focused therapy, functional or cognitive remediation, schema therapy, acceptance and commitment therapy, dialectical behaviour therapy, and other mindfulness-based therapies. Intervention formats will include online, telephone and face to face, delivered individually or in a group (or combinations of these). Selfmanagement interventions will be included, defined as any structured psychotherapeutic intervention accessed and completed wholly or partly by oneself, regardless of mode. For example, supported and unsupported online therapy programs, psychotherapeutic apps, and manualised therapies worked through independently will be included if they are formally assessed. Descriptions and details of all included interventions will be provided within the systematic review.

\section{Comparisons}

Studies must assess one or more psychosocial intervention/s alone or in combination. Descriptions and details of all included comparator interventions will be provided within the systematic review. Studies will not require a direct comparison between individuals at different putative stages of illness but must include analyses (or provide sufficient detail to draw this information from the study methodology and results) of a proxy measure of stage of illness.

\section{Outcomes}

For the present review, 'recovery-focused' primary outcomes will include outcomes in three categories: (i) general/social functioning (including functional impairment, daily general, social or occupational functioning, disability or autonomy), (ii) cognitive functioning or impairment and (iii) quality of life. Table 2 outlines the outcome measures to be included in this review.

\section{Study type}

RCTs and controlled trials will be sought. However, it is not anticipated that many RCTs will be available, especially for novel therapeutic modalities. Therefore, other intervention studies will be eligible for inclusion, including open pre-post trials and non-randomised controlled trials. Case series and case reports will be excluded due to the high risk of bias in these designs. Whilst broadening inclusion criteria invites the inclusion of studies with a higher risk of bias, this will be addressed with the implementation of risk of bias criteria and offset by the anticipated gains including the implications of review findings for future research and clinical practice.

\section{Procedures \\ Search strategy}

Scopus, PsychInfo, PubMed and Web of Science databases will be searched to identify potentially eligible studies for inclusion. No date restrictions will be applied and search terms will be broad to increase the likelihood of capturing potentially eligible studies. Unpublished trials, ongoing trials and grey literature will not be included as these are not anticipated to contribute to the fulfilment of the review aims. An example of the proposed search strategy for Scopus is outlined in Table 3.

\section{Quality and risk of bias}

Risk of bias within randomised trials will be assessed with the Cochrane Collaboration tool for assessing risk of bias [100], within non-randomised studies using the Risk Of Bias In Non-randomised Studies of Interventions (ROBINS-I) tool [101], and Grading of Recommendations Assessment, Development and Evaluation (GRADE) methodology across studies, with this assessment taken into consideration in any conclusions [102].

\section{Data extraction and management}

Following PRISMA-P guidelines, we will provide a flowchart to demonstrate each element of the search.

Relevance After deduplication, both raters (HT and $\mathrm{CM}$ ) will screen all titles and abstracts against inclusion criteria for relevance, specifically population, study type, interventions, and outcomes. The full text of results will be reviewed as necessary to ensure accurate categorisation. 
Table 2 Outcome measures for the proposed review

\begin{tabular}{|c|}
\hline \\
\hline $\begin{array}{l}\text { 1. General/social functioning } \\
\text { A multidimensional construct encompassing an individual's } \\
\text { capacity for independent living, occupational and educational } \\
\text { achievement, interpersonal relationships and recreation [55]. } \\
\text { Measures will include any validated self-report or clinician-rated, } \\
\text { real-world functioning or performance-based measure, including } \\
\text { the following: Global Assessment of Functioning (GAF) [56], } \\
\text { Functioning Assessment Short Test (FAST) [57], The clinician-rated } \\
\text { Longitudinal Interval Follow-up Evaluation-Range of Impaired } \\
\text { Functioning Tool (LIFE-RIFT) [58], World Health Organisation } \\
\text { Disability Assessment Schedule (WHODAS-2) [59], Sheehan } \\
\text { Disability Scale (SDS) [60], Social Functioning Scale (SFS) [61], } \\
\text { Life Functioning Questionnaire (LFQ) [62], USCD Performance- } \\
\text { Based Skills Assessment [63], Bipolar Disorder Functioning } \\
\text { Questionnaire [64], Social and Occupational Functioning } \\
\text { Assessment Scale (SOFAS) [65] }\end{array}$ \\
\hline $\begin{array}{l}\text { Cognitive functioning } \\
\text { An individual's range of abilities relating to cognition, memory } \\
\text { and learning, including attention, executive function, verbal } \\
\text { learning and memory, verbal fluency, processing speed, working } \\
\text { memory, visual learning and memory, psychomotor speed, } \\
\text { visuo-spatial ability) [66]. Measures will include any validated } \\
\text { measure or screening tool assessing cognitive function or } \\
\text { impairment overall or by domain including the following: Mini- } \\
\text { Mental State Exam [67], Mini-Cog [68], Memory Impairment Screen } \\
\text { (MIS) [69], General Practitioner Assessment of Cognition (GPCOG) } \\
\text { [70], The clock drawing test (CDT) [71], Montreal Cognitive } \\
\text { Assessment (MoCA) [72], FAST, cognitive domain [57], Weschler } \\
\text { Adult Intelligence Scale (WAIS-IV) or its subtests [73], Wechsler } \\
\text { Memory Scale (WMS-IV) or its subtests [74], Wide Range Assessment } \\
\text { of Memory and Learning [75], California Verbal Learning Test [76], } \\
\text { Hopkins Verbal Learning Test-Revised [77], Brief Visuospatial Memory } \\
\text { Test-Revised [78], Rey-Osterrieth Complex Figure Test [79], Trail } \\
\text { Making Test Part A or Part B [80], Wisconsin Card Sorting Test [81], } \\
\text { Delis-Kaplan Executive Function System [82], Boston Naming Test [83], } \\
\text { Controlled Oral Word Association [84], MATRICS Consensus Cognitive } \\
\text { Battery (MCCB) [85] }\end{array}$ \\
\hline $\begin{array}{l}\text { Quality of life } \\
\text { While quality of life is used in varying ways, the World Health } \\
\text { Organisation defines this as "an individual's perception of their } \\
\text { position in life in the context of the culture and value systems } \\
\text { in which they live and in relation to their goals, expectations, } \\
\text { standards and concerns" [86]. Measures will include any validated } \\
\text { self-report or clinician-rated, general or disorder specific measure } \\
\text { of quality of life, including the following: QoL.BD [87], EuroQoL [88], } \\
\text { Lancashire Quality of Life Profile [89]/Manchester Short Assessment } \\
\text { of Quality of Life (MANSA) [90], Lehman Quality of Life Interview [91], } \\
\text { Longitudinal Interval Follow-up Evaluation [92], MOS Short Form } \\
\text { 12[93],MOS Short Form } 20 \text { [94], MOS Short Form 36 [95], Quality of } \\
\text { Life Enjoyment and Satisfaction Questionnaire (Q-LES-Q) [96], Quality } \\
\text { of Life in Depression Scale [97], Quality of Life Index [98], World } \\
\text { Health Organisation Quality of Life Assessment [99] }\end{array}$ \\
\hline
\end{tabular}

Uncertainties regarding eligibility for inclusion will be resolved via consensus. Where multiple publications of the same study or from the same dataset exist, these will be narrowed to a single summary that reports key variables relevant to this review.

Extraction Data will be extracted independently by both raters from all included studies using a data extraction form developed for this review (Additional file 2). Both raters will pilot this form with five studies initially and
Table 3 Scopus search terms for the proposed review Search terms TITLE-ABS-KEY (bipolar OR mania OR manic* OR "manic depres**") AND TITLE-ABS-KEY ("psychological therap*" OR psychotherap* OR psychosocial OR "self-management" OR "Psychological intervention" OR cbt OR "cognitive therapy" OR "behavior therapy" OR "behavioural therapy" OR "cognitive behavioural" OR "cognitive behavior" OR mindfulness OR mbct OR "collaborative care" OR ipsrt OR "social rhythm" OR interpersonal OR "Acceptance and Commitment Therapy" OR "ACT" OR dbt OR "dialectical behaviour therapy" OR psychoeducation OR "family therapy" OR "family focused" OR carer OR "functional remediation" OR "cognitive remediation" OR "schema therapy") AND TITLE-ABS-KEY (stage* OR staging OR predict* OR mediat* OR moderat* OR neurocognit* OR cognit* OR comorbid* OR episodes OR "number of episodes" OR function* OR trajectory OR "illness history" $O R$ "illness characteristics" OR "course of illness") AND TITLE-ABS-KEY (recovery OR "patient-reported outcomes" OR "quality of life" OR function* OR employment OR autonomy OR wellbeing OR social OR disability OR meaning OR vocation* OR cognit* OR acceptance OR self-compassion)

make amendments if required prior to data extraction. Extracted data will include study design, sample size, study population, participant demographics (including diagnoses), baseline characteristics including details of stage-related proxy measures, details of the intervention, details of all reported study outcomes, and information required for assessment of the risk of bias. Where missing data are identified for variables relevant to this review, study authors will be contacted to request these. Studies will be excluded if authors do not provide these data.

\section{Data synthesis}

Data will be synthesised using narrative synthesis methods, as the broad aims and inclusion criteria will preclude meaningful statistical data pooling. A table of all the extracted data will be presented in the systematic review. A data-based convergent synthesis design will be employed [103], focusing on the interaction between interventions, stage of illness and outcomes. Participants' likely stage of illness will be identified based on the plausible proxy measures and outcomes grouped into 'cognitive, 'functioning, and 'quality of life'. Data-based convergent synthesis involves describing and synthesising results qualitatively, drawing out themes or categories. Data for each outcome type will be synthesised per putative stage of illness. Next, to address the second question, different intervention types will be examined.

\section{Discussion}

This review will constitute a significant addition to the literature regarding the psychological treatment of $\mathrm{BD}$, by applying the lens of 'staging of illness'. Findings will further illuminate the role of plausible proxy measures of stage of illness in the effectiveness of psychological interventions for established $\mathrm{BD}$, by synthesising the evidence regarding proxy measures of stage of illness 
moderating interventions for recovery-focused and functional outcomes.

There is a clear need for a summary of evidence relating to stage of illness in the treatment of established BD; however, reviews to date have focused on symptoms alone and a limited range of proxy measures for the staging construct $[25,50]$. The current review extends previous reviews in several ways. Most importantly, the range of outcomes which may be meaningful for those with BD will be explored, including examination of their applicability across stages of illness. This is essential in light of previous findings suggesting that different outcomes may be relevant at different stages of illness [32, 39]. For example, symptom relief and relapse prevention appear less amenable to intervention with advancing illness [22, 30,34] while quality of life and functioning have been effectively targeted later in the course of illness [32, 39]. Further, potential differences in effectiveness between interventions across putative stages of illness will be examined, as prior studies have suggested that particular intervention types have more relevance at different stages of illness [2, 22, 25, 32-34]. Broader eligibility criteria will enable the examination of other putative stage-related factors rather than relying solely on number of episodes as a proxy measure of stage of illness. Theoretically, a broader range of factors-such as functional impairment and neurocognitive functioning-may be relevant to stage of illness [29]. Such studies may provide new insights that were not available to prior reviews that were narrower in scope. If the review's two driving questions are answered in the affirmative, the project will provide an empirical platform for the identification, development and tailoring of novel psychological intervention strategies for individuals with $\mathrm{BD}$ based on stage of illness, ultimately providing a more focused direction for clinicians, consumers and researchers.

However, it should be noted that qualitative systematic reviews have a number of limitations. These include often involving less rigour than quantitative data syntheses and being subject to researchers' views and biases (e.g., [104]). We will attempt to minimise the impacts of these limitations through the rigorous methods described in this protocol. Another anticipated limitation is that few studies of any one intervention are expected, which may inhibit meaningful synthesis.

\section{Endnote}

${ }^{1} \mathrm{We}$ use the qualifier 'plausible' to remind the reader (and ourselves) that 'staging' remains a hypothetical construct in psychiatry. Any proxies are at best consistent with one or more of the various provisional conceptualisations of staging.

\section{Additional files}

Additional file 1: PRISMA-P 2015 Checklist. (DOCX $34 \mathrm{~kb}$ )

Additional file 2: DATA EXTRACTION FORM. (DOCX 14 kb)

\section{Abbreviations}

ACT: Acceptance and commitment therapy; BD I: Bipolar I disorder; BD II: Bipolar II disorder; BD: Bipolar disorder; BD-NOS: Bipolar disorder not otherwise specified; CBT: Cognitive-behavioural therapy; CM: Carla McEnery; DBT: Dialectical behaviour therapy; DSM: Diagnostic and Statistical Manual; FAST: Functioning Assessment Short Test; GAF: Global Assessment of Functioning; GM: Greg Murray; GRADE: Grading of Recommendations Assessment, Development and Evaluation; HT: Hailey Tremain;

ICD: International, Classification of Diseases; IPSRT: Interpersonal and Social Rhythm Therapy; JS: Jan Scott; KF: Kathryn Fletcher; LFQ: Life Functioning Questionnaire; LIFE-RIFT: Longitudinal Interval Follow-up Evaluation-Range of Impaired Functioning Tool; MB: Michael Berk; MBCT: Mindfulness-based cognitive therapy; PRISMA: Preferred Reporting Items for Systematic Reviews and Meta-Analyses; PRISMA-P: Preferred Reporting Items for Systematic Reviews and Meta-Analyses Protocol; PROSPERO: Prospective Register of Systematic Reviews; RCT: Randomised controlled trial; ROBINS-l: Risk Of Bias In Non-randomised Studies of Interventions; SDS: Sheehan Disability Scale; SOFAS: Social and Occupational Functioning Assessment Scale; STEPBD: Systematic Treatment Enhancement Program for Bipolar Disorder; TAU: Treatment as usual; WHODAS-2: World Health Organisation Disability Assessment Schedule

\section{Acknowledgements}

Not applicable.

\section{Funding}

The article processing charge was funded by the Centre for Mental Health, Swinburne University. These funders had no role in the study design, data collection and analysis, decision to publish, or preparation of the manuscript. HT is supported by an Australian Government Research Training Program Scholarship.GM receives funding from the National Health and Medical Research Council for a related project, grant number APP1102097. MB is supported by a NHMRC Senior Principal Research Fellowship (1059660). JS was a visiting fellow with Swinburne University.

\section{Availability of data and materials}

Not applicable.

\section{Authors' contributions}

HT and GM primarily contributed to the review concept and analysis plan. $H T, G M$ and KF had primary contributions to the preparation of the manuscript, while all authors were involved in the editing of the manuscript. All authors read and approved the final manuscript.

Ethics approval and consent to participate

Not applicable.

Consent for publication

Not applicable.

Competing interests

The authors declare that they have no competing interests.

\section{Publisher's Note}

Springer Nature remains neutral with regard to jurisdictional claims in published maps and institutional affiliations.

\section{Author details}

${ }^{1}$ Centre for Mental Health, Faculty of Health Arts and Design, Swinburne University, PO Box 218, John St Hawthorn VIC, Melbourne 3122, Australia. ${ }^{2}$ Academic Psychiatry, Institute of Neuroscience, Newcastle University, Newcastle, UK. ${ }^{3}$ IMPACT Strategic Research Centre, School of Medicine, Barwon Health, Deakin University, Geelong, VIC, Australia. ${ }^{4}$ Orygen, The National Centre of Excellence in Youth Mental Health, Parkville, Australia. 
${ }^{5}$ Centre for Youth Mental Health, The University of Melbourne, Melbourne, Australia. ${ }^{6}$ The Department of Psychiatry and the Florey Institute for Neuroscience and Mental Health, the University of Melbourne, Parkville, VIC, Australia.

Received: 7 August 2018 Accepted: 12 May 2019

Published online: 25 May 2019

\section{References}

1. Duffy A, Horrocks J, Doucette S, Keown-Stoneman C, McCloskey S, Grof P. The developmental trajectory of bipolar disorder. Br J Psychiatry. 2014 https://doi.org/10.1192/bjp.bp.113.126706.

2. Berk M, Berk L, Dodd S, Cotton S, Macneil C, Daglas R, Conus P, Bechdolf A, Moylan S, Malhi GS. Stage managing bipolar disorder. Bipolar Disord. 2014. https://doi.org/10.1111/bdi.12099.

3. Post RM, Fleming J, Kapczinski F. Neurobiological correlates of illness progression in the recurrent affective disorders. J Psychiatr Res. 2012. https://doi.org/10.1016/j.jpsychires.2012.02.004.

4. Vieta E, Reinares M, Rosa AR. Staging bipolar disorder. Neurotox Res. 2011. https://doi.org/10.1007/s12640-010-9197-8

5. Rosa AR, Magalhaes PV, Czepielewski L, Sulzbach MV, Goi PD, Vieta E, Gama CS, Kapczinski F. Clinical staging in bipolar disorder: focus on cognition and functioning. J Clin Psychiatry. 2014. https://doi.org/10.4088/JCP.13m08625.

6. Scott J, Leboyer M, Hickie I, Berk M, Kapczinski F, Frank E, Kupfer D, McGorry P. Clinical staging in psychiatry: a cross-cutting model of diagnosis with heuristic and practical value. Br J Psychiatry. 2013. https://doi.org/10.1192/ bjp.bp. 112.110858

7. McGorry PD, Nelson B, Goldstone S, Yung A. Clinical staging: a heuristic and practical strategy for new research and better health and social outcomes for psychotic and related mood disorders. Can J Psychiat-Rev Can Psychiat. 2010;55:486-97.

8. Berk M, Conus P, Lucas N, Hallam K, Malhi GS, Dodd S, Yatham LN, Yung A, McGorry P. Setting the stage: from prodrome to treatment resistance in bipolar disorder. Bipolar Disord. 2007. https://doi.org/10.1111/j.1399-5618. 2007.00484.X.

9. Kapczinski F, Dias W, Kauer-Sant'Anna M, Frey BN, Grassi-Oliveira R, Colom F, Berk M. Clinical implications of a staging model for bipolar disorders. Expert Rev Neurother. 2009. https://doi.org/10.1586/ern.09.31.

10. Alda M, Kapczinski F. Staging model raises fundamental questions about the nature of bipolar disorder. J Psychiatry Neurosci. 2016;41:291-3.

11. Frank E, Nimgaonkar VL, Phillips ML, Kupfer DJ. All the world's a (clinical) stage: rethinking bipolar disorder from a longitudinal perspective. Mol Psychiatry. 2015. https://doi.org/10.1038/mp.2014.71.

12. Fernandes BS, Berk M. Staging in bipolar disorder: one step closer to precision psychiatry. Rev Bras Psiquiatr. 2017. https://doi.org/10.1590/15164446-2017-3902.

13. Kapczinski F, Magalhaes PV, Balanza-Martinez V, Dias W, Frangou S, Gama CS, Gonzalez-Pinto A, Grande I, Ha K, Kauer-Sant'Anna M, et al. Staging systems in bipolar disorder: an International Society for Bipolar Disorders Task Force Report. Acta Psychiatr Scand. 2014. https:/doi.org/10.1111/acps.12305.

14. Deckersbach T, Peters AT, Sylvia LG, Gold AK, da Silva Magalhaes PV, Henry DB, Frank E, Otto MW, Berk M, Dougherty DD, et al. A cluster analytic approach to identifying predictors and moderators of psychosocial treatment for bipolar depression: results from STEP-BD. J Affect Disord. 2016. https://doi.org/10.1016/j.jad.2016.03.064.

15. Grande I, Magalhaes PV, Chendo I, Stertz L, Panizutti B, Colpo GD, Rosa AR, Gama CS, Kapczinski F, Vieta E. Staging bipolar disorder: clinical, biochemical, and functional correlates. Acta Psychiatr Scand. 2014. https:// doi.org/10.1111/acps. 12268

16. Kauer-Sant'Anna M, Kapczinski F, Andreazza AC, Bond DJ, Lam RW, Young $L T$, Yatham LN. Brain-derived neurotrophic factor and inflammatory markers in patients with early- vs. late-stage bipolar disorder. Int J Neuropsychopharmacol. 2009. https://doi.org/10.1017/S1461145708009310

17. Berk M, Hallam KT, McGorry PD. The potential utility of a staging model as a course specifier: a bipolar disorder perspective. J Affect Disord. 2007. https:// doi.org/10.1016/j.jad.2007.03.007.

18. McGorry PD, Yung AR, Phillips L, Yuen HP, Francey S, Cosgrave EM, Germano D, Bravin J, McDonald T, Blair A, et al. Randomized controlled trial of interventions designed to reduce the risk of progression to first-episode psychosis in a clinical sample with subthreshold symptoms. Arch Gen Psychiatry. 2002;59:921-8.
19. Berk M, Berk L, Udina M, Moylan S, Stafford L, Hallam K, Goldstone S, McGorry PD. Palliative models of care for later stages of mental disorder: maximizing recovery, maintaining hope, and building morale. Aust N Z J Psychiatry. 2012. https://doi.org/10.1177/0004867411432072.

20. Murray G, Leitan ND, Thomas N, Michalak EE, Johnson SL, Jones S, Perich T, Berk L, Berk M. Towards recovery-oriented psychosocial interventions for bipolar disorder: quality of life outcomes, stage-sensitive treatments, and mindfulness mechanisms. Clin Psychol Rev. 2017. https://doi.org/10.1016/j. cpr.2017.01.002.

21. Goi PD, Bucker J, Vianna-Sulzbach M, Rosa AR, Grande I, Chendo I, Sodre LA, Kauer-Sant'Anna M, Silveira L, Kunz M, et al. Pharmacological treatment and staging in bipolar disorder: evidence from clinical practice. Braz J Psychiatry. 2015. https://doi.org/10.1590/1516-4446-2014-1554.

22. Colom F, Reinares M, Pacchiarotti I, Popovic D, Mazzarini L, Martinez-Aran A, Torrent C, Rosa A, Palomino-Otiniano R, Franco C, et al. Has number of previous episodes any effect on response to group psychoeducation in bipolar patients? A 5-year follow-up post hoc analysis. Acta Neuropsychiatr. 2010. https://doi.org/10.1111/j.1601-5215.2010.00450.x.

23. Passos IC, Mwangi B, Vieta E, Berk M, Kapczinski F. Areas of controversy in neuroprogression in bipolar disorder. Acta Psychiatr Scand. 2016. https://doi. org/10.1111/acps.12581.

24. Berk M, Brnabic A, Dodd S, Kelin K, Tohen M, Malhi GS, Berk L, Conus P, McGorry PD. Does stage of illness impact treatment response in bipolar disorder? Empirical treatment data and their implication for the staging model and early intervention. Bipolar Disord. 2011. https://doi.org/10.1111/j. 1399-5618.2011.00889.x

25. Joyce K, Thompson A, Marwaha S. Is treatment for bipolar disorder more effective earlier in illness course? A comprehensive literature review. Int J Bipolar Disord. 2016. https://doi.org/10.1186/s40345-016-0060-6.

26. Lavagnino L, Cao B, Mwangi B, Wu MJ, Sanches M, Zunta-Soares GB, Kapczinski $F$, Soares J. Changes in the corpus callosum in women with late-stage bipolar disorder. Acta Psychiatr Scand. 2015. https://doi.org/10.1111/acps.12397.

27. Tremain $\mathrm{H}$, Fletcher $\mathrm{K}$, Murray $\mathrm{G}$. Number of episodes in bipolar disorder: the case for more thoughtful conceptualisation and measurement bipolar disorders (under review) 2019.

28. Nasrallah HA. Staging psychiatric disorders: a clinico-biologic model (from the editor). Curr Psychiatr Ther. 2013;12:9.

29. Reinares M, Papachristou E, Harvey P, Mar Bonnin C, Sanchez-Moreno J, Torrent C, Ayuso-Mateos JL, Ploubidis GB, Vieta E, Frangou S. Towards a clinical staging for bipolar disorder: defining patient subtypes based on functional outcome. J Affect Disord. 2013. https://doi.org/10.1016/j.jad.2012.06.005.

30. Reinares M, Colom F, Rosa AR, Bonnin CM, Franco C, Sole B, Kapczinski F, Vieta E. The impact of staging bipolar disorder on treatment outcome of family psychoeducation. J Affect Disord. 2010. https://doi.org/10.1016/j.jad.2009.09.009.

31. Andreazza AC, Kapczinski F, Kauer-Sant'Anna M, Walz JC, Bond DJ, Goncalves CA, Young LT, Yatham LN. 3-nitrotyrosine and glutathione antioxidant system in patients in the early and late stages of bipolar disorder. J Psychiatry Neurosci. 2009;34:263-71.

32. Murray G, Leitan ND, Berk M, Thomas N, Michalak E, Berk L, Johnson SL, Jones S, Perich T, Allen NB, et al. Online mindfulness-based intervention for late-stage bipolar disorder: pilot evidence for feasibility and effectiveness. J Affect Disord. 2015. https://doi.org/10.1016/j.jad.2015.02.024.

33. Miziou S, Tsitsipa E, Moysidou S, Karavelas V, Dimelis D, Polyzoidou V, Fountoulakis KN. Psychosocial treatment and interventions for bipolar disorder: a systematic review. Ann General Psychiatry. 2015. https://doi.org/ 10.1186/s12991-015-0057-z.

34. Scott J, Paykel E, Morriss R, Bentall R, Kinderman P, Johnson T, Abbott R, Hayhurst H. Cognitive-behavioural therapy for severe and recurrent bipolar disorders: randomised controlled trial. Br J Psychiatry. 2006. https://doi.org/ 10.1192/bjp.188.4.313.

35. Colom F, Vieta E, Martinez-Aran A, Reinares M, Goikolea JM, Benabarre A, Torrent C, Comes M, Corbella B, Parramon G, et al. A randomized trial on the efficacy of group psychoeducation in the prophylaxis of recurrences in bipolar patients whose disease is in remission. Arch Gen Psychiatry. 2003. https://doi.org/10.1001/archpsyc.60.4.402.

36. Reinares M, Colom F, Sanchez-Moreno J, Torrent C, Martinez-Aran A, Comes M, Goikolea JM, Benabarre A, Salamero M, Vieta E. Impact of caregiver group psychoeducation on the course and outcome of bipolar patients in remission: a randomized controlled trial. Bipolar Disord. 2008. https://doi. org/10.1111/j.1399-5618.2008.00588.x. 
37. Peters AT, Sylvia LG, Magalhaes PV, Miklowitz DJ, Frank E, Otto MW, Hansen NS, Dougherty DD, Berk M, Nierenberg AA, et al. Age at onset, course of illness and response to psychotherapy in bipolar disorder: results from the Systematic Treatment Enhancement Program For Bipolar Disorder (STEPBD). Psychol Med. 2014. https://doi.org/10.1017/S0033291714000804.

38. Murray G. You say you want a revolution: recovery, biomedicine and muddling through. Aust N Z J Psychiatry. 2015. https://doi.org/10.1177/0004867415610200.

39. Bonnin CM, Torrent C, Arango C, Amann BL, Sole B, Gonzalez-Pinto A, Crespo JM, Tabares-Seisdedos R, Reinares M, Ayuso-Mateos $\mathrm{L} L$, et al. Functional remediation in bipolar disorder: 1-year follow-up of neurocognitive and functional outcome. Br J Psychiatry. 2016. https://doi.org/10.1192/bjp.bp.114.162123.

40. Torrent C, Bonnin Cdel M, Martinez-Aran A, Valle J, Amann BL, Gonzalez-Pinto A, Crespo JM, Ibanez A, Garcia-Portilla MP, Tabares-Seisdedos R, et al. Efficacy of functional remediation in bipolar disorder: a multicenter randomized controlled study. Am J Psychiatry. 2013. https://doi.org/10.1176/appi.ajp.2012.12070971.

41. de Barros Pellegrinelli K, de OCLF, Silval KI, Dias W, Roso MC, Bandeira M, Colom F, Moreno RA. Efficacy of psychoeducation on symptomatic and functional recovery in bipolar disorder. Acta Psychiatr Scand. 2013. https:// doi.org/10.1111/acps.12007.

42. Smith DJ, Griffiths E, Poole R, di Florio A, Barnes E, Kelly MJ, Craddock N, Hood K, Simpson S. Beating bipolar: exploratory trial of a novel internetbased psychoeducational treatment for bipolar disorder. Bipolar Disord. 2011. https://doi.org/10.1111/j.1399-5618.2011.00949.x.

43. Yatham LN, Kennedy SH, Parikh SV, Schaffer A, Bond DJ, Frey BN, Sharma V, Goldstein BI, Rej S, Beaulieu S, et al. Canadian Network for Mood and Anxiety Treatments (CANMAT) and International Society for Bipolar Disorders (ISBD) 2018 guidelines for the management of patients with bipolar disorder. Bipolar Disord. 2018. https://doi.org/10.1111/bdi.12609.

44. Geddes JR, Miklowitz DJ. Treatment of bipolar disorder. Lancet (London, England). 2013. https://doi.org/10.1016/S0140-6736(13)60857-0.

45. Goodwin GM, Haddad PM, Ferrier IN, Aronson JK, Barnes T, Cipriani A, Coghill DR, Fazel S, Geddes JR, Grunze H, et al. Evidence-based guidelines for treating bipolar disorder: revised third edition recommendations from the British Association for Psychopharmacology. J Psychopharmacol. 2016. https://doi.org/10.1177/0269881116636545.

46. Oud M, Mayo-Wilson E, Braidwood R, Schulte P, Jones SH, Morriss R, Kupka R, Cuijpers P, Kendall T. Psychological interventions for adults with bipolar disorder: systematic review and meta-analysis. Br J Psychiatry. 2016. https:// doi.org/10.1192/bjp.bp.114.157123.

47. Salcedo S, Gold AK, Sheikh S, Marcus PH, Nierenberg AA, Deckersbach T, Sylvia LG. Empirically supported psychosocial interventions for bipolar disorder: current state of the research. J Affect Disord. 2016. https://doi.org/ 10.1016/j.jad.2016.05.018.

48. Reinares M, Sanchez-Moreno J, Fountoulakis KN. Psychosocial interventions in bipolar disorder: what, for whom, and when. J Affect Disord. 2014. https://doi.org/10.1016/j.jad.2013.12.017.

49. Murray G. Adjunctive psychosocial interventions for bipolar disorder: some psychotherapeutic context for the Canadian Network for Mood and Anxiety Treatments (CANMAT) \& International Society for Bipolar Disorders (ISBD) guidelines. Bipolar Disord. 2018. https://doi.org/10.1111/bdi.12655.

50. Lam DH, Burbeck R, Wright K, Pilling S. Psychological therapies in bipolar disorder: the effect of illness history on relapse prevention - a systematic review (report). Bipolar Disord. 2009;11:474.

51. Kupfer DJ, Frank E, Ritchey FC. Staging bipolar disorder: what data and what models are needed? Lancet Psychiatry. 2015; https://doi.org/10.1016/S22150366(15)00096-6.

52. Shamseer L, Moher D, Clarke M, Ghersi D, Liberati A, Petticrew M, Shekelle $P$, Stewart LA. Preferred reporting items for systematic review and metaanalysis protocols (PRISMA-P) 2015: elaboration and explanation. BMJ. 2015. https://doi.org/10.1136/bmj.g7647.

53. Liberati A, Altman DG, Tetzlaff J, Mulrow C, Gøtzsche PC, loannidis JPA, Clarke M, Devereaux PJ, Kleijnen J, Moher D. The PRISMA statement for reporting systematic reviews and meta-analyses of studies that evaluate health care interventions: explanation and elaboration. J Clin Epidemiol. 2009. https://doi.org/10.1016/j.jclinepi.2009.06.006.

54. Faedda GL, Baldessarini RJ, Glovinsky IP, Austin NB. Pediatric bipolar disorder: phenomenology and course of illness. Bipolar Disord. 2004;6:305-13.

55. Levy B, Medina AM, Weiss RD. Cognitive and psychosocial functioning in bipolar disorder with and without psychosis during early remission from an acute mood episode: a comparative longitudinal study. Compr Psychiatry. 2013. https://doi. org/10.1016/j.comppsych.2012.12.018.
56. Jones SH, Thornicroft G, Coffey M, Dunn G. A brief mental health outcome scale: reliability and validity of the global assessment of functioning (gaf). Br J Psychiatry. 1995;166:654-9.

57. Rosa AR, Sanchez-Moreno J, Martinez-Aran A, Salamero M, Torrent C, Reinares M, Comes M, Colom F, Van Riel W, Ayuso-Mateos JL, et al. Validity and reliability of the functioning assessment short test (fast) in bipolar disorder. Clin Pract Epidemiol Ment Health. 2007. https:/doi.org/10.1186/1745-0179-3-5.

58. Leon A, Solomon D, Mueller T, Turvey C, Endicott J, Keller M. The range of impaired functioning tool (life-rift): a brief measure of functional impairment. Psychol Med. 1999;29:869-78.

59. Organization WH. World Health Organization disabilty assessment schedule: WHODAS II. Phase 2 field trials. Health services research. 2000.

60. Arbuckle R, Frye MA, Brecher M, Paulsson B, Rajagopalan K, Palmer S, Degl'Innocenti A. The psychometric validation of the Sheehan Disability Scale (SDS) in patients with bipolar disorder. Psychiatry Res. 2009;165:163-74.

61. Birchwood M, Smith J, Cochrane R, Wetton S, Copestake S. The social functioning scale the development and validation of a new scale of social adjustment for use in family intervention programmes with schizophrenic patients. Br J Psychiatry. 1990;157:853-9.

62. Altshuler L, Mintz J, Leight K. The Life Functioning Questionnaire (LFQ): a brief, gender-neutral scale assessing functional outcome. Psychiatry Res. 2002. https://doi.org/10.1016/S0165-1781(02)00180-4.

63. Patterson TL, Goldman S, McKibbin CL, Hughs T, Jeste DV. UCSD performancebased skills assessment: development of a new measure of everyday functioning for severely mentally ill adults. Schizophr Bull. 2001;27:235-45.

64. Aydemır Ö, Eren İ, Savaş H, Oğuzhanoğlu NK, Kocal N, Özgüven HD, Akkaya C, Başterzı AD, Karlidağ R, Yenılmez Ç. Development of a questionnaire to assess inter-episode functioning in bipolar disorder: bipolar disorder functioning questionnaire. Turk J Psychiatry. 2007;18.

65. Rybarczyk B. Social and occupational functioning assessment scale (SOFAS). Encyclopedia of clinical neuropsychology; 2011. p. 2313.

66. Burdick KE, Ketter TA, Goldberg JF, Calabrese JR. Assessing cognitive function in bipolar disorder: challenges and recommendations for clinical trial design. J Clin Psych. 2015;76:e342.

67. Folstein MF, Folstein SE, McHugh PR. "Mini-mental state": a practical method for grading the cognitive state of patients for the clinician. J Psychiatr Res. 1975;12:189-98.

68. Borson S, Scanlan J, Brush M, Vitaliano P, Dokmak A. The mini-cog: a cognitive 'vital signs' measure for dementia screening in multi-lingual elderly. Int J Geriatr Psychiatry. 2000;15:1021-7.

69. Buschke H, Kuslansky G, Katz M, Stewart WF, Sliwinski MJ, Eckholdt HM, Lipton RB. Screening for dementia with the memory impairment screen. Neurology. 1999;52:231.

70. Brodaty H, Pond D, Kemp NM, Luscombe G, Harding L, Berman K, Huppert FA. The gpcog: a new screening test for dementia designed for general practice. J Am Geriatr Soc. 2002;50:530-4.

71. Shulman Kl. Clock-drawing: is it the ideal cognitive screening test? Int J Geriatr Psychiatry. 2000;15:548-61.

72. Nasreddine ZS, Phillips NA, Bédirian V, Charbonneau S, Whitehead V, Collin I, Cummings JL, Chertkow H. The Montreal cognitive assessment, Moca: a brief screening tool for mild cognitive impairment. J Am Geriatr Soc. 2005;53:695-9.

73. Wechsler D. Wechsler adult intelligence scale-fourth edition (WAIS-IV), vol. 22. San Antonio, TX: NCS Pearson; 2008. p. 498.

74. Wechsler D. Wechsler memory scale iv (wms-iv). In.: New York. NY: Psychological Corporation; 2009.

75. Sheslow D, Adams W. Wide Range Assessment Of Memory And LearningSecond Edition (WRAML2). Delaware: Wide range Inc; 2003.

76. Woods SP, Delis DC, Scott JC, Kramer JH, Holdnack JA. The California Verbal Learning Test-second edition: test-retest reliability, practice effects, and reliable change indices for the standard and alternate forms. Arch Clin Neuropsychol. 2006;21:413-20.

77. Benedict RH, Schretlen D, Groninger L, Brandt J. Hopkins Verbal Learning Test-Revised: normative data and analysis of inter-form and test-retest reliability. Clin Neuropsychol. 1998;12:43-55.

78. Benedict RH, Schretlen D, Groninger L, Dobraski M, Shpritz B. Revision of the brief visuospatial memory test: studies of normal performance, reliability, and validity. Psychol Assess. 1996:8:145.

79. Rey A. L'examen psychologique dans les cas d'encéphalopathie traumatique.(les problems.). Arch Psychol (Geneve). 1941.

80. Gordon NG. The trail making test in neuropsychological diagnosis. J Clin Psychol. 1972 
81. Heaton RK. Wisconsin card sorting test manual; revised and expanded. Psychol Assess Resour. 1981:5-57.

82. Delis DC, Kramer JH, Kaplan E, Holdnack J. Reliability and validity of the Delis-Kaplan executive function system: an update. J Int Neuropsychol Soc. 2004;10:301-3.

83. Goodglass H, Kaplan E, Weintraub S: Boston naming test: Lea \& Febiger; 1983.

84. Ruff R, Light R, Parker S, Levin H. Benton controlled oral word association test: reliability and updated norms. Arch Clin Neuropsychol. 1996:11:329-38.

85. Kern RS, Gold JM, Dickinson D, Green MF, Nuechterlein KH, Baade LE, Keefe RS, Mesholam-Gately RI, Seidman LJ, Lee C. The MCCB impairment profile for schizophrenia outpatients: results from the matrics psychometric and standardization study. Schizophr Res. 2011;126:124-31.

86. WHOQOL Group. The World Health Organization Quality Of Life Assessment (WHOQOL): position paper from the world health organization. Soc Sci Med. 1995:41:1403-9.

87. Michalak EE, Murray G, RTtSPliBD C. Development of the QoL.BD: a disorderspecific scale to assess quality of life in bipolar disorder. Bipolar Disord. 2010. https://doi.org/10.1111/j.1399-5618.2010.00865.x.

88. Rabin R, Fd C. EQ-SD: a measure of health status from the EuroQol group. Ann Med. 2001;33:337-43.

89. Van Nieuwenhuizen C, Schene A, Koeter M, Huxley P. The Lancashire quality of life profile: modification and psychometric evaluation. Soc Psychiatry Psychiatr Epidemiol. 2001;36:36-44.

90. Priebe S, Huxley P, Knight S, Evans S. Application and results of the Manchester short assessment of quality of life (Mansa). Int J Soc Psychiatry. 1999;45:7-12

91. Russo J, Roy-Byrne P, Reeder D, Alexander M, DWYER-O'CONNOR ED, Dagadakis C, Ries R, Patrick D. Longitudinal assessment of quality of life in acute psychiatric inpatients: reliability and validity. J Nerv Ment Dis. 1997; 185:166-75.

92. Keller MB, Lavori PW, Friedman B, Nielsen E, Endicott J, McDonald-Scott P, Andreasen NC. The longitudinal interval follow-up evaluation. A comprehensive method for assessing outcome in prospective longitudinal studies. Arch Gen Psychiatry. 1987. https://doi.org/10.1001/archpsyc.1987. 01800180050009.

93. Ware JE Jr, Kosinski M, Keller SD. A 12-item short-form health survey: construction of scales and preliminary tests of reliability and validity. Med Care. 1996:220-33.

94. Ware JE, Sherbourne CD, Davies AR. Developing and testing the MOS 20item short-form health survey. 1992

95. McHorney CA, Ware JE Jr, Lu JR, Sherbourne CD. The MOS 36-item ShortForm Health Survey (SF-36): III. Tests of data quality, scaling assumptions, and reliability across diverse patient groups. Med Care. 1994:40-66.

96. Endicott J, Nee J, Harrison W, Blumenthal R. Quality of life enjoyment and satisfaction questionnaire: a new measure. Psychopharmacol Bull. 1993;29(2): 321-6. https://medworksmedia.com/journals/psychopharmacology-bulletin/.

97. Hunt SM, McKenna SP. The QLDS: a scale for the measurement of quality of life in depression. Health Policy. 1992;22:307-19.

98. Ferrans CE, Powers MJ. Quality of life index: development and psychometric properties. Adv Nurs Sci. 1985.

99. Group TW. The World Health Organization Quality Of Life Assessment (WHOQOL): development and general psychometric properties. Soc Sci Med. 1998;46:1569-85.

100. Higgins JPT, Altman DG, Gotzsche PC, Juni P, Moher D, Oxman AD, Savovic J, Schulz KF, Weeks L, Sterne JAC. The Cochrane Collaboration's tool for assessing risk of bias in randomised trials. BMJ. 2011. https://doi.org/10. 1136/bmj.d5928.

101. Sterne JAC, Hernán MA, Reeves BC, Savović J, Berkman ND, Viswanathan M, Henry D, Altman DG, Ansari MT, Boutron I, et al. ROBINS-I: a tool for assessing risk of bias in non-randomised studies of interventions. BMJ. 2016 https://doi.org/10.1136/bmj.i4919.

102. Guyatt GH, Oxman AD, Vist GE, Kunz R, Falck-Ytter Y, Alonso-Coello P Schünemann HJ. GRADE: an emerging consensus on rating quality of evidence and strength of recommendations. BMJ. 2008;336:924.

103. Hong QN, Pluye P, Bujold M, Wassef M. Convergent and sequential synthesis designs: implications for conducting and reporting systematic reviews of qualitative and quantitative evidence. Systematic reviews. 2017. https://doi.org/10.1186/s13643-017-0454-2.

104. Dixon-Woods M, Bonas S, Booth A, Jones DR, Miller T, Sutton AJ, Shaw RL, Smith JA, Young B. How can systematic reviews incorporate qualitative research? A critical perspective. Qual Res. 2006;6:27-44.

Ready to submit your research? Choose BMC and benefit from:

- fast, convenient online submission

- thorough peer review by experienced researchers in your field

- rapid publication on acceptance

- support for research data, including large and complex data types

- gold Open Access which fosters wider collaboration and increased citations

- maximum visibility for your research: over $100 \mathrm{M}$ website views per year

At BMC, research is always in progress.

Learn more biomedcentral.com/submissions 